\title{
Simplified 2-D Analytical Model for Winding Loss Analysis of Flyback Transformers
}

\author{
Junming Zhang ${ }^{\dagger}$, Wei Yuan ${ }^{\star}$, Hulong Zeng ${ }^{\star}$, and Zhaoming Qian ${ }^{\star}$ \\ ${ }^{\dagger \star}$ College of Electrical Engineering, Zhejiang University, Hangzhou, China
}

\begin{abstract}
The winding loss analysis of a flyback transformer is difficult and ambiguous because the primary side current and the secondary side current differs both in shape and phase, especially for DCM (Discontinuous Conduction Mode) operation. Meanwhile, the fringing field caused by the air gaps further makes the traditional 1-D loss analysis model not directly applicable. The paper gives a thorough investigation into the phase shift of winding currents, which indicates that the phase shift of the high order harmonics is still close to $180^{\circ}$ out-of-phase. Based on the analysis, a simplified 2-D winding loss analytical model for flyback transformers considering the effects of low order harmonics is proposed. By neglecting the $y$ components of the fringing field, the proposed model has an acceptable accuracy and a simple form that is similar to the conventional 1-D model. The power loss calculated with the proposed analysis model is verified by FEA (Finite Element Analysis) simulations and experimental results.
\end{abstract}

Key words: DCM, Flyback, Winding loss

\section{NOMENCLATURE}

$I_{p}$ : Peak value of the real primary side current

$I_{s}$ : Peak value of the real secondary side current

$D_{1}$ : Duty when primary side conducts current

$D_{2}$ : Duty when secondary side conducts current

$D_{\mathrm{r}}$ : Duty of the transition period

$T$ : Period of the current excitation

$b_{w}$ : Winding width

$H$ : Magnetic field strength

$m_{p}$ : Total primary layer number

$m_{s}$ : Total secondary layer number

$a$ : Primary layer number, counting from left to right

$b:$ Secondary layer number, counting from left to right

$i$ : Harmonic number, counting from 0 to $n$

$n$ :Total harmonic counted

$I_{p}(i)$ : Peak current value of the $i$-th harmonic in one primary layer

$I_{s}(i)$ : Peak current value of the $i$-th harmonic in one secondary layer

$\varphi_{p}(i): i$-th harmonic phase of primary current

$\varphi_{s}(i): i$-th harmonic phase of secondary current

Manuscript received Sep. 1, 2011; revised Sep. 3, 2012

Recommended for publication by Associate Editor Honnyong Cha.

${ }^{\dagger}$ Corresponding Author: zhangjm@zju.edu.cn

Tel: +86-571-8795-3095, Fax: +86-571-8795-2224, Zhejiang University

${ }^{*}$ College of Electrical Engineering, Zhejiang University, China
$H_{y}(a, b)_{i}$ : Magnetic field at $i$-th harmonic in the region outer side of layer $(a, b)$ by the proximity effect in y axis $H_{\mathrm{gc}}(a, b)_{i}$ : Center gap fringing field of $i$-th harmonic in layer $(a, b)$

$H_{\mathrm{gs}}(a, b)_{i}$ : Spacer gap fringing field of $\mathrm{i}$-th harmonic in layer $(a, b)$

$l(a, b)$ : Length of the Layer(a,b)

$H_{\text {gcy }}$ : y component of center gap fringing field $H_{g c}$

$H_{\text {gcx }}$ : x component of center gap fringing field $H_{g c}$

$H_{\text {gsy }}:$ y component of the spacer gap fringing field $H_{\mathrm{gs}}$

$H_{\mathrm{gsx}}$ : x component of the spacer gap fringing field $H_{\mathrm{gs}}$

$x_{c}(a, b)_{\mathrm{k}}$ : distance from wire $\mathrm{k}$ in layer( $\left.\mathrm{a}, \mathrm{b}\right)$ to center gap in $\mathrm{x}$-axis

$y_{c}(a, b)_{\mathrm{k}}$ : distance from wire $\mathrm{k}$ in layer $(\mathrm{a}, \mathrm{b})$ to center gap in $\mathrm{y}$-axis

$x_{s}(a, b)_{\mathrm{k}}$ : distance from wire $\mathrm{k}$ in layer( $\left.\mathrm{a}, \mathrm{b}\right)$ to spacer gap in $\mathrm{x}$-axis

$y_{s}(a, b)_{\mathrm{k}}$ : distance from wire $\mathrm{k}$ in layer $(\mathrm{a}, \mathrm{b})$ to spacer gap in $\mathrm{y}$-axis

$l_{g}$ : Gap length

$l_{m}$ : Core magnetic path length

$j: \sqrt{-1}$

$J_{y}:$ Electric current density $\left(\mathrm{A} / \mathrm{m}^{2}\right)$

$\varphi(i)$ : Phase shift at $i$-th harmonic

$\omega(i)$ : Angular frequency of the $i$-th harmonic

$\eta$ :Layer copper factor 
$\mu_{0}$ : Permeability of free space

$\mu$ : Relative Permeability of the core

$\sigma$ : Conductivity, $\sigma=5.8 \times 10^{7}(\Omega \cdot \mathrm{m})^{-1}$

$\delta$ : Skin depth, $\delta=\sqrt{2 / 2 \pi f \mu_{o} \sigma}$

$Q_{y}$ : The proximity effect loss per square meter

$Q_{g c}$ : The center gap effect loss per square meter

$Q_{g s}:$ The side gap effect loss per square meter

$P_{\mathrm{y}}$ : The proximity effect loss

$P_{\mathrm{g}}$ : The gap effect loss

$P_{\text {tot }}$ : Total loss including proximity and gap effect loss

$D$ : Diameter of the round wires

$d$ : Thickness of the equivalent foil

$N$ : Wire number in one layer

$F_{r}:$ AC resistance factor $R_{a c} / R_{d c}$

$R_{d c \_}$: DC resistance of the primary side

$R_{d c_{-}}:$DC resistance of the secondary side

\section{INTRODUCTION}

The conduction loss of transformer windings increases significantly with the switching frequency due to the eddy current loss caused by the skin effect and the proximity effect. The eddy current loss has been studied in many applications based on the 1-D model proposed by Dowell [1]-[5]. For non-sinusoidal current waveforms, which are quite common in switch mode power supplies, Fourier decomposition is usually applied to get the harmonic components, the losses caused by each harmonic are then summed up to get the total power loss since the harmonic components are orthogonal [6], [7]. These methods could be further simplified by series expansions and applied to any periodic waveform [8]. The winding loss for a flyback transformer using this 1-D model and the Fourier decomposition method was also introduced in [28]-[30].

However, these methods are not accurate or proper for winding loss analysis of flyback type transformers. Firstly, the current flowing in both sides is not simultaneous, when non-sinusoidal waveforms are decomposed into Fourier components, the harmonic field on either side of each layer can not be easily obtained because the phase shift of the two currents is not $180^{\circ}$ out of phase. Secondary, the magnetizing current is not negligible because an air gap is usually used in a ferrite core, which results in 2-D fields which make the traditional 1-D loss model inaccurate.

The conventional way for flyback transformer winding loss analysis is by using 2-D or even 3-D finite element models in the time domain. By modeling the off-state foil as an open circuit and ensuring that the on-state winding has a continuous, steady state sinusoidal current, a substantial circulating current in the off-state foil winding was reported in [9]. It is absolutely correct in recognizing the proximity effects introducing a significant loss in the off-state winding. However, the main drawback of this method is the neglecting of the transition period when current flows through both sides simultaneously, which contributes a large part of the total winding loss by the time domain FEA models [10]-[12]. The SFD (Squared Field Derivative) method gives a more accurate and computational efficient winding loss evaluation method considering the 2-D effect [12], [13]. However, it is still based on a computer-aided process like FEA and needs complex geometric modeling and parameter configuration, which is usually time consuming and difficult for power supply design engineers.

Another method for winding loss analysis is based on frequency domain analysis. Mutual resistance was introduced in [14] to get a general representation of the eddy current loss in a two winding transformer. The averaged power loss was expressed by the complex conjugate of the two sinusoidal winding currents, which was also reported in [15] and represented in a different form. Though these methods implied a general way to analyze the power loss in windings containing currents with a phase shift, how to evaluate the resistance matrix in a 2-D model considering the fringing field when the total MMF (Magnetomotive Force) is not zero has still not been presented. Paper [16] proposed a winding loss analytical model by decomposing the total winding current into two components: the transformer current and the inductor current. This is verified to be quite accurate in the CCM (Continuous Conduction Mode). But it is not explicit for DCM because the orthogonal behavior between the transformer current and the inductor current is not valid anymore.

This paper focuses on flyback transformer winding loss analysis in DCM operation. First, the phase shift between the primary and secondary winding currents is analyzed in the frequency domain. The relationship between the current phase shift and the 2-D air gap effect are also discussed. Then, the precondition and the accuracy of the previous 1-D mutual resistance method to analyze flyback transformer winding loss are clarified. Based on the characteristic of the proximity effect and the fringing field between windings, a simplified 2-D winding loss analytical model for a flyback transformer in the frequency domain is proposed. The ac winding losses of the flyback transformer in DCM is calculated by the proposed method, which is verified by FEA simulations and experimental results. In addition, the proposed method can also be used to explain the inherent reason that the winding structure has an important effect on the winding loss of flyback transformers.

\section{KEY FACTOR OF THE WINDING LOSS ANALYSIS OF FLYBACK TRANSFORMERS}

Before a detailed analysis on the winding loss of flyback transformers is made, some important concepts and key aspects for winding loss analysis, which differ from conventional transformer winding loss analysis, are discussed here.

A. Current Excitation of the Flyback Transformer 


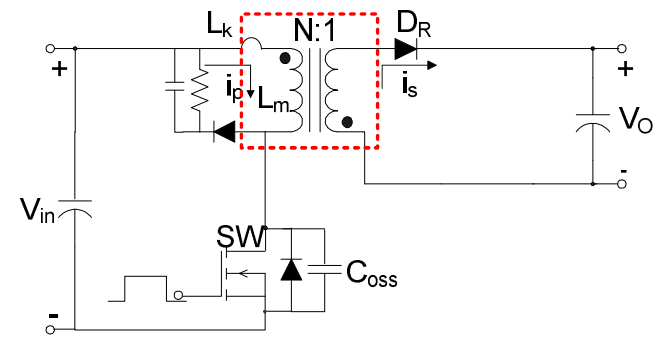

(a) Flyback topology.

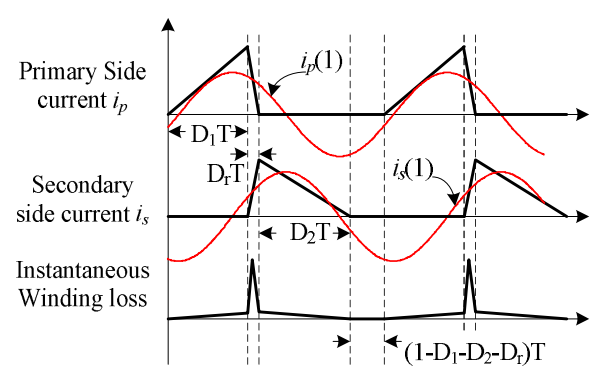

(b) Current waveforms under DCM mode.

Fig. 1. Flyback topology and current waveforms under DCM.

The flyback topology is shown in Fig. 1(a). The current for DCM is shown in Fig 1(b), which can be divided into four periods. The first period is $D_{I} T$, when the primary side switch is on. The second one is the transition period $D_{r} T$, when the energy starts to transfer from the primary side to the secondary side. During this period, the primary side current, $I_{p}$, falls and the secondary side current, $I_{s}$, rises, and both windings conduct current. The third period is $D_{2} T$, when the secondary winding conducts current. The last period is the idle period $\left(1-D_{1}-D_{r}-D_{2}\right) \mathrm{T}$ when neither of the windings conducts current.

Based on the current waveform described above, by using Fourier decomposition in the frequency domain, the amplitude and the phase of each harmonics current can be derived. Detailed expressions are given in Appendix A. Unlike forward type transformers, the phase shift of each harmonic in a flyback transformer is not $180^{\circ}$ out of phase, which makes the winding loss analysis more complex. Fig. 1(b) also shows the fundamental components of the primary side winding current and the secondary side winding current based on Fourier decomposition, i.e. $i_{p}(1)$ and $i_{s}(1)$. It is clear that they are not exactly $180^{\circ}$ out of phase.

Once the transformer primary side and secondary side currents are not exactly $180^{\circ}$ out of phase, the total MMF is not zero anymore. If the phase shift between the $i$-th primary side harmonic current, $i_{p}(i)$, and secondary side harmonic current, $i_{s}(i)$, is $\varphi(i)$, as shown in Fig. 2(a), the total MMF is not zero, as shown in Fig. 2(b). Therefore, the fringing magnetic field strays outside of the air gap, which will cause a 2-D effect on the magnetic field. For the traditional 1-D transformer winding loss calculation method, the current phase shift should be $180^{\circ}$

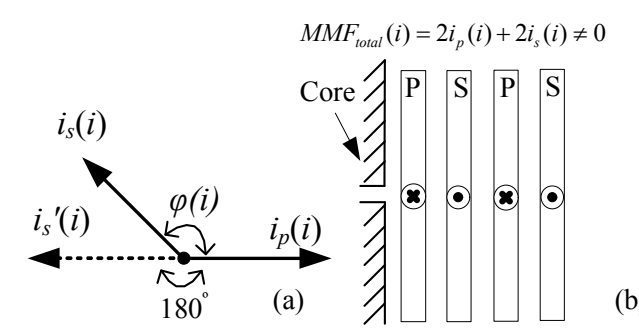

Fig. 2. (a) Phase shift angle of the primary and secondary current at $i$-th harmonic (b) transformer geometry and total MMF.

out of phase to keep the total MMF zero, such as $i_{p}(i)$ and $i_{s}^{\prime}(i)$ shown in Fig. 2(a). Therefore, in the flyback transformer under DCM operation, the traditional 1-D method can not be directly used for analysis considering the 2-D effect.

\section{B. The Relationship between Phase Shift and Air Gap Fringing Field}

As mentioned above, the gap fringing field usually exists when the total MMF is not zero. The gap fringing field and its effect on winding loss have been thoroughly analyzed in inductors with lumped or distributed air gaps [17]-[21], and it is concluded that the gap fringing field is strongly affected by the field at the center of the gap $\left(H_{g c}\right)$, the total gap length and the distance from the winding to the gap. The field at the center of the gap with total $2 l_{g}$ gap length based on Ampere's law is given as:

$$
H_{g c}=\frac{M M F_{\text {total }}}{2 l_{\mathrm{g}}+l_{m} / \mu}
$$

When the total MMF is zero as in the traditional 1-D model (this means the winding currents are $180^{\circ}$ out of phase), the gap fringing field is also zero as shown in Fig. 3(a). Therefore, the high frequency winding loss is mainly caused by the skin effect and the proximity effect. If the current phase shift differs from $180^{\circ}$, the total MMF will increase, and the gap fringing field intensity will increase too. Fig. 3(b) shows the gap fringing filed when the phase shift between the two winding currents is $225^{\circ}$, and both the gap and the proximity effect affect the winding loss, which makes the traditional 1-D analysis method not applicable or accurate. When the windings are properly kept away from the fringing field, the high frequency winding loss is still dominated by the proximity effect.

\section{Phase Shift of the Flyback Transformer Currents}

Theoretically, the phase shift of the winding currents is $180^{\circ}$ for a conventional transformer and $0^{\circ}$ for a pure inductor. The flyback transformer can be treated as a combination of a conventional transformer and an inductor. That means the phase shift can be different and depends on the combination itself, which is the physical meaning of this current phase shift in a flyback transformer. Since the phase shift of the winding 


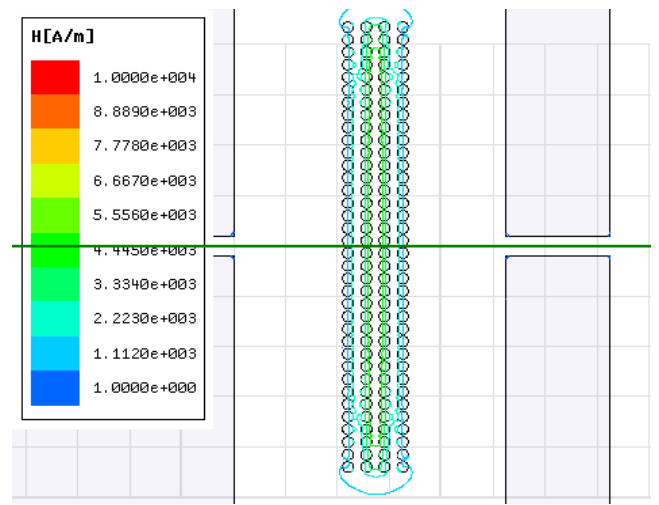

(a) Phase shift is $180^{\circ}$.

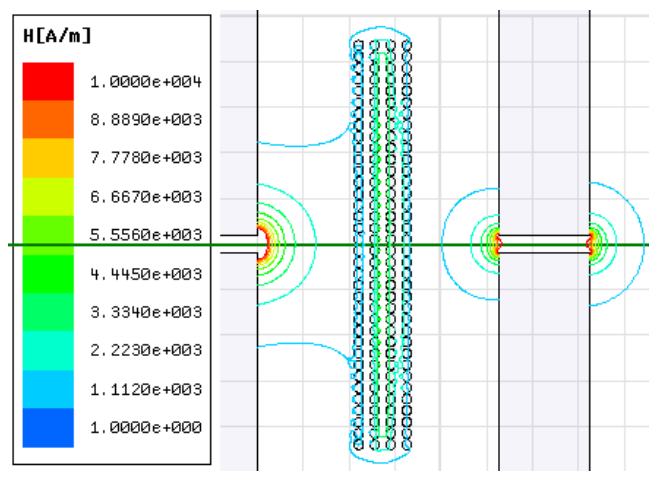

(b) Phase shift is $225^{\circ}$.

Fig. 3. Gap fringing field in a transformer with 2 primary winding layer and 2 secondary winding layer (p-p-s-s), air gap $1_{\mathrm{g}}=0.4 \mathrm{~mm}$, $\mathrm{I}_{\mathrm{p}}=\mathrm{I}_{\mathrm{s}}=1 \mathrm{~A}$ (sinusoidal peak current), $\mathrm{f}_{\mathrm{s}}=100 \mathrm{kHz}$. Ferrite core.

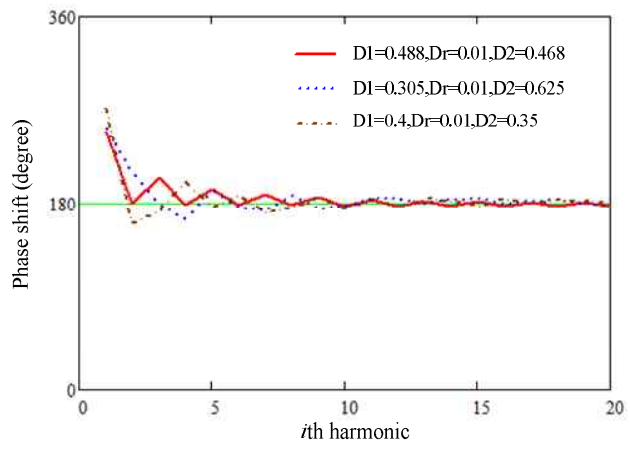

Fig. 4. The phase shift of the winding currents in the flyback transformer under DCM operation.

currents will affect the winding loss, it is important to investigate the phase shift of each current harmonic in a flyback transformer.

The flyback transformer winding current in DCM mode is shown in Fig. 1(b). Using Fourier decomposition in the frequency domain, the amplitude and the phase of each harmonics current can be derived. The phase shift between each harmonic can also be obtained. For simplicity, detailed expressions for the amplitude and phase of each winding current harmonic are given in Appendix A.

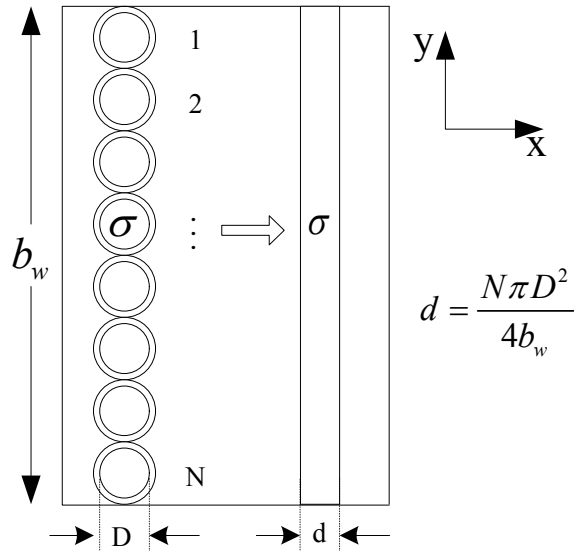

Fig. 5. Proposed equivalent expression of round wires.

The calculated results with different parameters using (A.9) are shown in Fig. 4. From Fig. 4, it is clear that the phase shift at higher harmonics ( $i \geq 2$ ) is close to $180^{\circ}$. As mentioned above, the fringing field is related to the phase shift. Therefore, in a flyback transformer, the gap effect is quite smaller when compared to the proximity effect for high order harmonics because the phase shift is close to $180^{\circ}$. The traditional 1-D loss model may still be effective and applicable in the frequency domain, and the 1-D mutual resistance method can be applied if the gap effect at the $1^{\text {st }}$ harmonic is much smaller when compared to the proximity effect.

\section{Layer Copper Factor for Round Wires}

Round wires are widely used in transformer windings. How to get high frequency eddy current loss in round wires in a simple way is also important. Usually a "layer copper factor" $\eta$ is used to take the layer porosity into account by inducing the $\eta$ factor to modify the conductivity, $\sigma$, and consequently the skin depth, $\delta$ [3]-[5], [8], [15]. However, this $\eta$ factor has been proven to be erroneous and can not be justified mathematically or physically in 1-D theories, and it can only be taken as a purely empirical definition [22]. In addition, the accuracy of this traditional equivalence is not good when the current phase is far away from $180^{\circ}$ (in section IV).

In this paper, a simple and straightforward equivalent method to equalize the porous layer to the nonporous layer is adopted. Fig. 5 shows the proposed expression for the round wires, where the effective copper area is kept the same without inducing the $\eta$ factor to modify conductivity or skin depth. This new equivalent thickness is then used to get the ac resistance factor $F_{r}$. The calculation result and the FEA simulation results will be presented in Section IV.

\section{SimPlified 2-D LOSS ANALYTICAL MODEL FOR FLYBACK TRANSFORMERS}

As mentioned above, the precondition to adopting a 1-D model in a winding loss analysis is to make the fringing field 


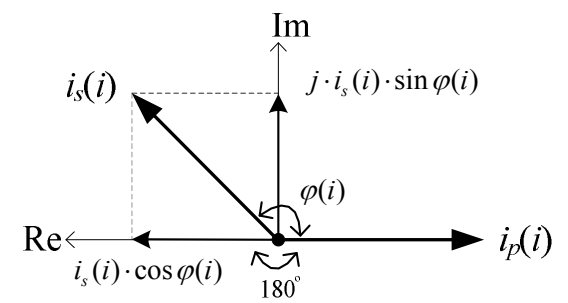

Fig. 6. Secondary current decomposition by the phase shift.

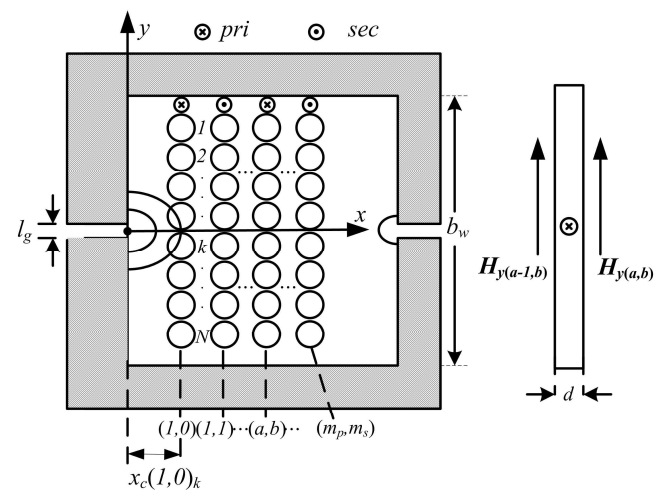

Fig. 7. The cross section of gapped core with winding.

much smaller than the proximity effect field at the $1^{\text {st }}$ harmonic. Though the gap fringing field in inductors with single winding was presented in [23], the gap field of the flyback transformer is quite different and strongly related to the current phase shift in the two windings. To clearly illustrate the effect of the phase shift on the fringing field and the proximity effect, the secondary current is decomposed into two parts: the real part, which is $180^{\circ}$ out of phase to the primary current, and the imaginary part, which is orthogonal to the real part, as shown in Fig. 6.

When the phase shift is applied to get the total MMF, the $x$ and $y$ components of the $i$-th harmonic fringing field are given in (2) and (3) as derived in [23]. Then the $i$-th harmonic fringing field at the edge of the center gap $([\mathrm{x}, \mathrm{y}]=[0,0]$, as shown in Fig. 7) can be derived in (4).

$$
\begin{aligned}
& H_{g c x}\left(x_{c}, y_{c}\right)_{i}=\frac{H_{g c}(i)}{2 \pi} \ln \left[\frac{x_{c}^{2}+\left(y_{c}-l_{\mathrm{g}} / 2\right)^{2}}{x_{c}^{2}+\left(y_{c}+l_{\mathrm{g}} / 2\right)^{2}}\right] \\
& H_{g c y}\left(x_{c}, y_{c}\right)_{i}=\frac{H_{g c}(i)}{\pi}\left\{\tan ^{-1}\left[\frac{x_{c} l_{\mathrm{g}}}{x_{c}{ }^{2}+y_{c}{ }^{2}-\left(l_{\mathrm{g}} / 2\right)^{2}}\right]+u_{g} \pi\right\} \\
& H_{g c}(i)=0.9 \frac{\left|m_{p} \cdot I_{p}(i)+m_{s} \cdot I_{s}(i) \cdot \cos (\varphi(i))+j \cdot m_{s} \cdot I_{s}(i) \cdot \sin (\varphi(i))\right|}{2 l_{\mathrm{g}}+l_{m} / \mu} \\
& \approx 0.9 \frac{\left|m_{p} \cdot I_{p}(i)+m_{s} \cdot I_{s}(i) \cdot \cos (\varphi(i))+j \cdot m_{s} \cdot I_{s}(i) \cdot \sin (\varphi(i))\right|}{2 l_{\mathrm{g}}}
\end{aligned}
$$

where $x_{c}$ and $y_{c}$ are the distances from the wire to the center of the gap in $\mathrm{x}$ and $\mathrm{y}$ axis, $u_{g}=0$ if $x_{c}{ }^{2}+y_{c}{ }^{2}-\left(l_{g} / 2\right)^{2} \geq 0$, and $u_{g}=1$ if $x_{c}^{2}+y_{c}^{2}-\left(l_{g} / 2\right)^{2}<0$.
In the model shown in Fig. 7, each layer is composed of round conductors with a diameter close to the skin depth, $\delta$, to minimize the skin effect. Hence the losses mainly considered here are the proximity effect loss and the gap fringing loss. Assuming the curvature of the winding layer is quite small, each layer is then represented as infinitely high and long. In addition, the gaps are placed in both the center post and the spacer to facilitate the experimental comparison. Therefore, the $y$ component of the fringing field is in the opposite direction for the center and spacer gaps field, which consequently cancels out the fringing effect in the $y$ axis [24].

For round conductors, the fringing field does not decay significantly like the foil layer due to their small size in all dimensions when compared with the winding window dimension. However, it still decays with distance or when it crosses a layer, which means the fringing field in the layer adjacent to the gap is the largest one when compared to other layers [18], [25], [26]. Hence it is reasonable to consider the fringing field loss only in the layer closest to the gap in the flyback transformer.

For the fringing field, the $x$ component is orthogonal to the proximity magnetic field and the $y$ component is parallel to the proximity magnetic field. The two components vary with the distance from the wire to the gap in the $\mathrm{x}$ and $\mathrm{y}$ axis. The loss caused by the $x$ component can be calculated directly due to its orthogonal behavior with respect to the proximity magnetic field. However, it is difficult and complex to get the loss caused by the $y$ component for many wires in a layer in the 2-D condition. To simplify the field loss calculation, if the averaged $y$ component of the fringing field of the layer adjacent to the gap is much smaller than the 1-D magnetic field of the $1^{\text {st }}$ harmonic, the $y$ component of the fringing field can be neglected for simplicity, as given in (5).

$\left|\overline{H_{g y}(1,0)_{1}}\right|=\frac{\sum_{k=1}^{N}\left|H_{g c y}\left(x_{c}(1,0)_{k}, y_{c}(1,0)_{k}\right)_{1}-H_{g s y}\left(x_{s}(1,0)_{k}, y_{s}(1,0)_{k}\right)_{1}\right|}{N}<<\left|H_{y}(1,0)_{1}\right|$

Here $\overline{H_{g y}(1,0)_{1}}$ is the averaged $y$ component of the fringing field in layer $(1,0)$ of the $1^{\text {st }}$ harmonic, which consists of the center and side gap field, and layer $(1,0)$ is the layer next to the center gap. $H_{g c y}\left(x_{c}(1,0)_{k}, y_{c}(1,0)_{k}\right)_{1}$ is the $y$ component of the center gap fringing field of wire $\mathrm{k}$ of the $1^{\text {st }}$ harmonic in the layer( 1,0$)$, and $H_{g s y}\left(\mathrm{x}_{\mathrm{s}}(1,0)_{\mathrm{k}}, \mathrm{y}_{\mathrm{s}}(1,0)_{\mathrm{k}}\right)_{1}$ is the $y$ component of the side gap fringing field of wire $\mathrm{k}$ of the $1^{\text {st }}$ harmonic in the layer $(1,0)$. The distances $x_{c}(1,0)_{k}$ and $x_{s}(1,0)_{k}$ are constant for each wire in layer( $(1,0)$, while $y_{c}(1,0)_{k}$ and $y_{s}(1,0)_{k}$ are related to the wire position in the layer. Fig. 8 shows a comparison of the averaged y component of the fringing field and the 1-D magnetic field in the layer near the gap based on the structure and current excitation shown in Fig.3. This implies that when the phase shift is close to $180^{\circ}$, the $y$ component of the fringing field $\left(H_{g y}\right)$ is negligible when 


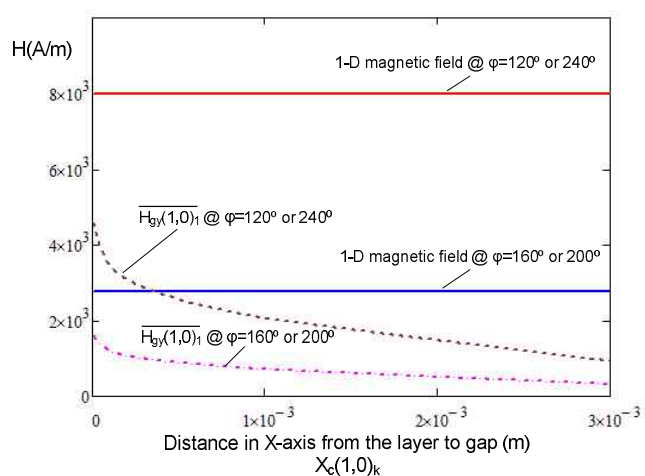

Fig .8. Averaged y component of the fringing field vs. the 1-D magnetic field in the layer near the gap.

compared to the 1-D magnetic field. Meanwhile, when the phase shift differs from $180^{\circ}, H_{g y}$ can also be neglected by placing the winding at a proper distance from the gap, which is usually true in practical applications due to the thickness of the insulation or transformer bobbin. With a $1.15 \mathrm{~mm}$ distance (close to the thickness of a bobbin), the averaged $H_{g y}$ is only one fourth of the 1-D magnetic field even with a $240^{\circ}$ phase shift.

By neglecting the $y$ component, the fringing field loss of each wire in the layers adjacent to the gap could be expressed as (6) considering the $x$ component, which is orthogonal to the proximity magnetic field. The fringing loss per square meter of the $i$-th harmonic at the layer next to the spacer gap can be obtained in the same way, as given in (7). It should be noted that the critical precondition for (6) and (7) is that the wire diameter be in the same order to or smaller than the skin depth.

$$
\begin{aligned}
Q_{g c}(1,0)_{i} & =\sum_{k=1}^{k=N} \frac{\sigma l D^{4} \omega(i)^{2}}{128}\left|H_{g c x}\left(x_{c}(1,0)_{k}, y_{c}(1,0)_{k}\right)_{i}\right|^{2} \\
Q_{g s}\left(m_{p}, m_{s}\right)_{i} & =\sum_{k=1}^{k=N} \frac{\sigma l D^{4} \omega(i)^{2}}{128}\left|H_{g s x}\left(x_{s}\left(m_{p}, m_{s}\right)_{k}, y_{s}\left(m_{p}, m_{s}\right)_{k}\right)_{i}\right|^{2}
\end{aligned}
$$

The 2-D field loss is simplified with only two parts in the flyback winding, one is the fringing loss from its $x$ component and the other is the proximity effect loss which can be deduced from the 1-D analytical model. How to get the magnetic field around each layer is important since the total MMF is not zero. Assume that the distance from the layer $(1,0)$ to the center gap is smaller than that from the outer layer $\left(\mathrm{m}_{\mathrm{p}}, \mathrm{m}_{\mathrm{s}}\right)$ to the side gap, and that the field at right side of the layer $\left(\mathrm{m}_{\mathrm{p}}, \mathrm{m}_{\mathrm{s}}\right)$ is close to zero in the model shown in Fig. 7 , which is usually true in practical applications. The assumption is more intuitive if there is only a center gap [26]. The magnetic field for each layer can be calculated from the outer layer to the inner layer.

With the decomposition of the secondary current, the complex type magnetic field of the $i$-th harmonic current in the right side of winding layer $(a, b)$ can be deduced based on the 1-D field analysis as follows:

$H_{y}(a, b)_{i}=\frac{\left(m_{p}-a\right) \cdot I_{p}(i)+\left(m_{p}-b\right) \cdot I_{s}(i) \cdot \cos (\varphi(i))+j \cdot\left(m_{p}-b\right) \cdot I_{s}(i) \cdot \sin (\varphi(i))}{b_{w}}(8)$

where $a / b$ are the numbers of primary/secondary layers included in the region as shown in Fig.7, which is determined by the winding structure. The field at the left side of the layer can be expressed in (9) for the primary layer and (10) for the secondary layer.

$H_{y}(a-1, b)_{i}=\frac{\left(m_{p}-a+1\right) \cdot I_{p}(i)+\left(m_{s}-b\right) \cdot I_{s}(i) \cdot \cos (\varphi(i))+j \cdot\left(m_{s}-b\right) \cdot I_{s}(i) \cdot \sin (\varphi(i))}{b_{w}}(9)$
$H_{y}(a, b-1)_{i}=\frac{\left(m_{p}-a\right) \cdot I_{p}(i)+\left(m_{s}-b+1\right) \cdot I_{s}(i) \cdot \cos (\varphi(i))+j \cdot\left(m_{s}-b+1\right) \cdot I_{s}(i) \cdot \sin (\varphi(i))}{b_{w}}(10)$

With the field given in (8), (9) or (10), the current density of the $i$-th harmonic for the primary side layer(a,b) as a function of $x$ is given as:

$$
\begin{aligned}
& J_{y}(a, b, x)_{i}=\frac{-k(i)}{\sinh \left(k(i) \cdot d_{f}\right)}\left[H_{y}(a, b)_{i} \cdot \cosh [k(i) \cdot x]\right. \\
& \left.-H_{y}(a-1, b)_{i} \cdot \cosh [k(i) \cdot(d-x)]\right]
\end{aligned}
$$

where:

$$
k(i)=\sqrt{j \omega(i) u_{o} \sigma}
$$

And the power dissipated per square meter by the proximity effect for this layer is given as:

$$
Q_{y}(a, b)_{i}=\frac{1}{\sigma} \cdot \int_{0}^{d}\left(\left|J_{y}(a, b, x)_{i}\right|\right)^{2} d x
$$

The power loss caused by the proximity effect in layer(a,b) can then be expressed as (14).

$$
P_{y}(a, b)_{i}=b w \cdot l(a, b) \cdot Q_{y}(a, b)_{i}
$$

Then the total winding loss considering the eddy effect and the gap fringing effect can be easily derived based on (6), (7) and (13), which is given in (15).

$$
\begin{aligned}
P_{t o t}=P_{y}+P_{g}=\sum_{i=0}^{n}\left[\sum_{(a, b)=(1,0)}^{(m p, m s)} b_{w} l(a, b) Q_{y}(a, b)_{i}+b_{w} l(1,0) Q_{g c}(1,0)_{i}\right] \\
+b_{w} l\left(m_{p}, m_{s}\right) Q_{g s}\left(m_{p}, m_{s}\right)_{i}
\end{aligned}
$$

Only the gap fringing loss of the layer next to gap is considered here. The fringing loss of other layers can also be added if the fringing loss is larger or the $\eta$ factor is small. For $i=0$ (DC component), there is no high frequency effect, and the winding loss can be directly obtained from $I_{p}(0)^{2} \cdot R_{d c \_}+$ 
$I_{s}(0)^{2} \cdot R_{d c_{c} s}$. The expression is similar to the 1-D model without considering the fringing effect.

\section{Simulation VerificAtion}

Maxwell 2D simulation software is used to verify the analysis results using the proposed loss calculation method with different winding current phase shifts and real flyback winding currents.

\section{A. Accuracy of the AC Resistance Factor considering Phase Shift}

The relationship between the phase shift and the gap fringing field has been discussed in section II. Although the phase is close to $180^{\circ}$ for high order harmonics, the phase shift of low harmonics is not negligible $(i<2)$, which is usually more important than the high order harmonics due to its large amplitude. Therefore, determining how the phase shift affects the AC resistance factor $F_{r}$ (the ratio of the winding $\mathrm{AC}$ resistance to the $\mathrm{DC}$ resistance) would be an important step for the proposed method.

For the round conductor equivalence method mentioned in section II, firstly, the proposed equivalence method and the conventional equivalence method need to be compared with the FEA results when the phase shift is $180^{\circ}$ to justify the accuracy in the 1-D condition without the fringing field. The winding structure shown in Fig.3 is used for the comparison. The conventional layer factor equivalence method evaluated here is the same as equation (10) in [5]. This method has been proven to be reasonably accurate when the wire diameter is in the same order as the skin depth, while overestimating when the wire diameter is several times larger than the skin depth.

In AC resistance factor $F_{r}$ calculation, different frequency sinusoidal current excitation is applied to both windings. The $\mathrm{AC}$ winding loss can be calculated based on (8) to (14) with $\varphi(i)=180^{\circ}$. Then the AC resistance can be derived based on this $\mathrm{AC}$ power loss, and the $\mathrm{AC}$ resistance factor $F_{r}$ can be calculated based on the $\mathrm{AC}$ resistance and the $\mathrm{DC}$ resistance.

The calculated and simulated results are shown in Fig.9. Both the conventional and the proposed equivalence have reasonable accuracy when $\mathrm{d} / \delta$ is below 1 , while the proposed equivalence has a better accuracy when $\mathrm{d} / \delta$ is high, which improves the accuracy for high order harmonics.

When the phase shift moves away from $180^{\circ}, F_{r}$ also varies. Fig. 10 shows the calculated values of $F_{r}$ with the two equivalence methods, which are compared to the FEA simulation result when the phase shift varies from $90^{\circ}$ to $270^{\circ}$. The results illustrate that the $\mathrm{AC}$ resistance increases when the phase shift moves away form $180^{\circ}$. It also illustrates that the traditional equivalence method overestimates the loss, especially when the phase shift is large, while the proposed equivalence method has an acceptable accuracy when compared to the FEA simulation results. In addition, it

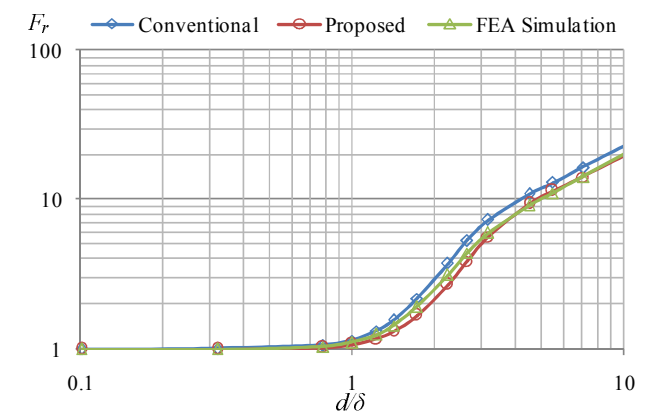

Fig. 9. Comparison of the AC resistor factor $F_{r} @ \mathrm{~d}=0.21 \mathrm{~mm}$, $\eta=0.74$, sweep frequency from $1 \mathrm{kHz}$ to $10 \mathrm{MHz}(\mathrm{d} / \delta=0.1-10)$, sinusoidal current excitations for the two windings with same peak value and 180 phase shift

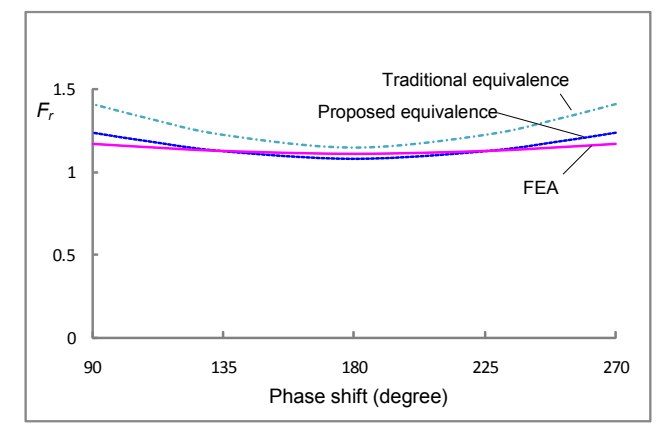

Fig. 10. Calculated and simulated AC resistance factor vs. phase shift@ $100 \mathrm{kHz}, \mathrm{d} / \delta=1$

verifies the effectiveness of the proposed equivalence method.

\section{B. Winding Loss with a Different Winding Structure.}

It was reported that the interleaving structure of a flyback transformer has very little effect on the winding loss reduction when compared to the forward type transformer [9]. This conclusion is made based on the assumption that the total winding loss is the sum of the primary side and secondary side winding losses. This means that the losses in the transition period are also calculated separately and consequently lead to larger winding losses. Thus it overestimates the loss of the interleaving structure.

The mechanism of the interleaving structure in winding loss reduction is that the MMF across the layer can be canceled out by different windings when the current in each winding is out of phase, as shown in Fig. 11. Using Ampere's law, the field at the boundary of the layer with the interleaving structure is much smaller than that with the non-interleaving structure. Consequently, it has a smaller winding loss.

Theoretically, interleaving is effective in reducing the proximity loss when the phase shift is from $90^{\circ}$ to $270^{\circ}$ according to (8). Considering the phase shift analyzed in section II, it is clear that the interleaving structure is still 


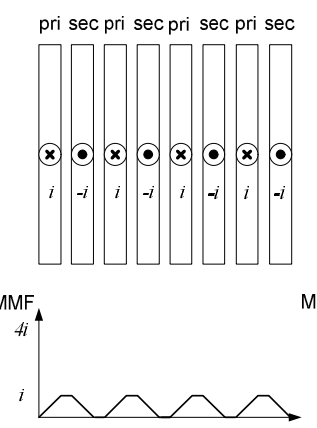

(a)
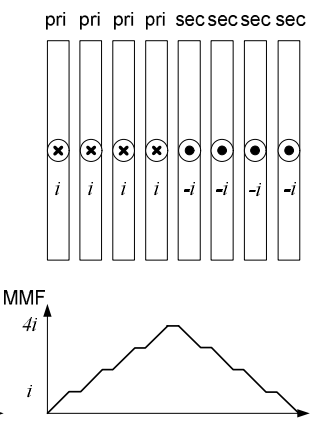

(b)
Fig. 11. MMF of the layers with (a) Interleaving. (b) Non-interleaving.

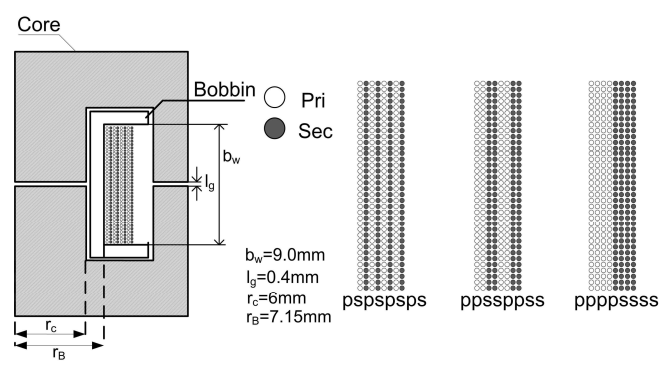

Fig. 12. 2-D winding structure of the simulated transformers. (a) pspspsps. (b) ppssppss. (c)ppppssss.

TABLE I

PARAMETERS OF THE TRANSFORMERS WITH DifFERENT WIRES

\begin{tabular}{|c|c|c|}
\hline $\begin{array}{c}\text { Winding } \\
\text { specification }\end{array}$ & D=0.21 mm(AWG32) & $\mathrm{D}=\mathbf{0 . 3 1 m m}(\mathrm{AWG} 28)$ \\
\hline Turn Ratio & $48: 8$ & $48: 8$ \\
\hline $\begin{array}{c}\text { Primary side } \\
\text { winding }\end{array}$ & $\begin{array}{c}\text { 4 layers,36 wires per } \\
\text { layer(12*3) }\end{array}$ & $\begin{array}{c}\text { 4 layers,24 wires per } \\
\text { layer(12*2) }\end{array}$ \\
\hline $\begin{array}{c}\text { Secondary side } \\
\text { winding }\end{array}$ & $\begin{array}{c}\text { 4 layers,32 wires per } \\
\text { layer( } 2 * 16)\end{array}$ & $\begin{array}{c}\text { 4 layers,24 wires per } \\
\text { layer( }(2 * 12)\end{array}$ \\
\hline Core & Geomitry:PQ2620, Material:PC44, MnZn Ferrite \\
\hline Gap length $\left(1_{\mathrm{g}}\right)$ & \multicolumn{2}{|c|}{ Both $0.4 \mathrm{~mm}$ at center and outer post } \\
\hline $\begin{array}{c}\text { Distance from } \\
\text { gap to layer }\end{array}$ & $\begin{array}{c}\mathrm{xc}(1,0)_{\mathrm{k}}=1.2 \mathrm{~mm}, \\
\mathrm{xs}(4,4)_{\mathrm{k}}=1.75 \mathrm{~mm}\end{array}$ & $\begin{array}{c}\mathrm{xc}(1,0)_{\mathrm{k}}=1.2 \mathrm{~mm}, \\
\mathrm{xs}(4,4)_{\mathrm{k}}=1.2 \mathrm{~mm}\end{array}$ \\
\hline $\begin{array}{c}\text { Current } \\
\text { excitation }\end{array}$ & $\mathrm{D}_{1}=0.488, \mathrm{Dr}=0.01, \mathrm{D}_{2}=0.468, \mathrm{I}_{\mathrm{p}}=3.03 \mathrm{~A}, \mathrm{I}_{\mathrm{s}}=18.18 \mathrm{~A}$ \\
\hline
\end{tabular}

effective for flyback transformers since the phase shift between most of windings current harmonics is close to $180^{\circ}$.

The three winding structures shown in Fig. 12 are calculated and simulated to verify the effectiveness of the proposed method with real current waveforms in the flyback windings. They are also used to evaluate the loss difference between the different winding structures. Two kinds of round wires are adopted for the windings with very similar conductor spacing factors, $\eta$, to study the influences of the wire diameter. Detailed parameters of the transformer are given in Table I.

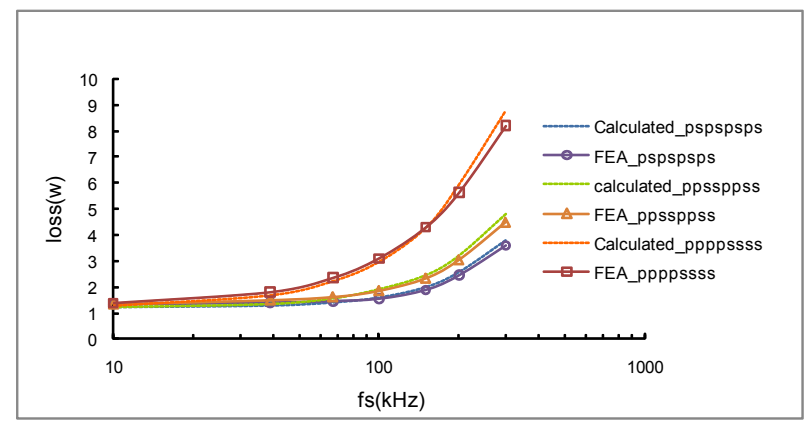

(a)

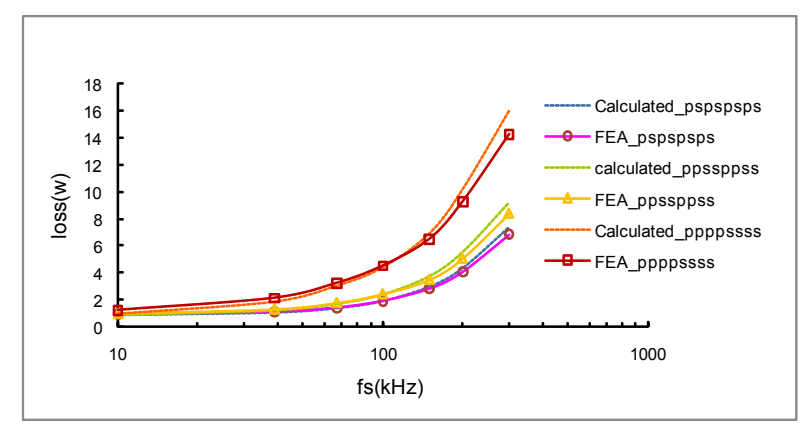

(b)

Fig. 13. Loss comparison with different structures and wire diameter. (a) $\mathrm{D}=0.21 \mathrm{~mm}$. (b) $\mathrm{D}=0.31 \mathrm{~mm}$.

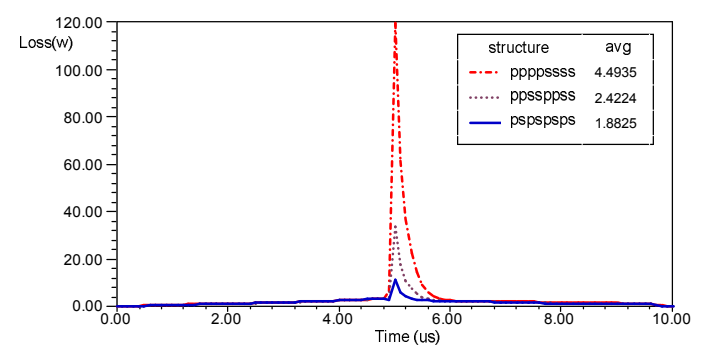

Fig. 14. Simulated winding loss with different structures in time domain with current excitation given in Table I.

The calculated results using the proposed method (up to the $15^{\text {th }}$ harmonics, $\mathrm{i}=15$ ) are shown in Fig. 13 with different wire diameters. A detailed example for loss calculation with the proposed method is given in Appendix B. The calculated results are also compared to the FEA time domain simulation results. The time step is 50ns in the simulation to get a satisfactory accuracy. From Fig. 13, the proposed loss model has a very good accuracy in loss evaluation when compared with the FEA time domain simulation results.

There is a significant loss difference with the different winding structures which shows that the phase shifted currents are still effective in reducing the winding loss with a proper structure. Generally, with an equal layer current amplitude, the winding loss with interleaving structure becomes smaller when the phase shift is close to $180^{\circ}$. Therefore, the power loss caused by high frequency 
harmonics is reduced in the interleaving structure since their phase shift is close to $180^{\circ}$. The power loss difference with the different winding structures is mainly caused by the transition period $\left(D_{r} T\right)$ where the currents in both windings change quickly and simultaneously with a reversed $\mathrm{d} i / \mathrm{d} t$. This is also verified by the time domain FEA simulation results, as shown in Fig. 14.

\section{Wire Diameter Optimization}

Base on the proposed analytical model, wire diameter optimization can be achieved by a calculation instead of a complex FEA simulation. Fig.15 shows a magnet wire optimization example using the proposed model with two winding structures based on the current waveform specified in Table 1 at different switching frequencies $(39 \mathrm{kHz}, 66 \mathrm{kHz}$, and $100 \mathrm{kHz}$, respectively). From Fig. 15, the optimal wire diameter for an interleaving (pspspsps) structure can be a little larger than that for a non-interleaving (pppppsss) structure due to smaller eddy current losses. The optimization of the round conductors can be obtained by (16) based on the results of the equivalent foil layers as shown in Fig. 5. For example, the optimal foil thickness at $39 \mathrm{kHz}$ in the interleaving structure is $0.17 \mathrm{~mm}$. Thus the optimal diameter of the round conductor is about $0.29 \mathrm{~mm}$.

$$
D=\sqrt{\frac{4 \cdot b_{w} \cdot d}{\pi \cdot N}}
$$

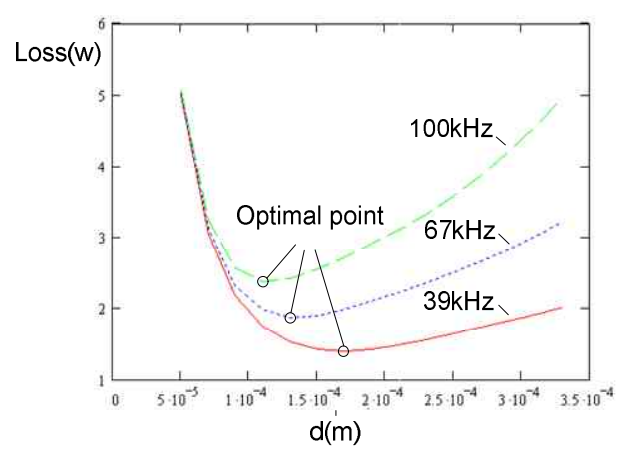

(a) pspspsps structure

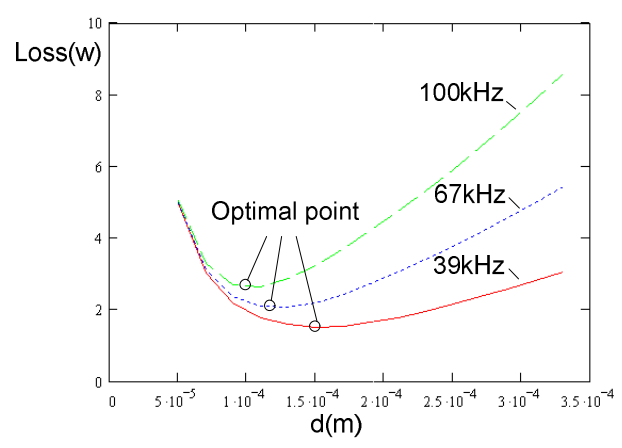

(b) ppppssss structure

Fig. 15. Wire diameter optimization at different switching frequency and winding structure.

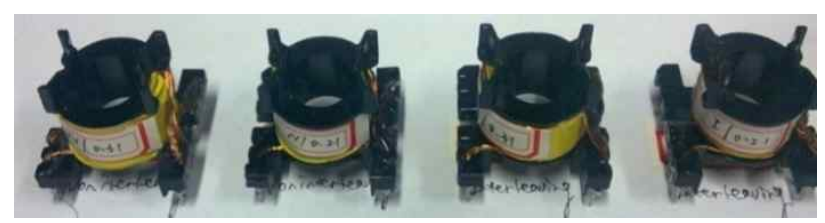

Fig. 16. Picture of the four tested transformers.

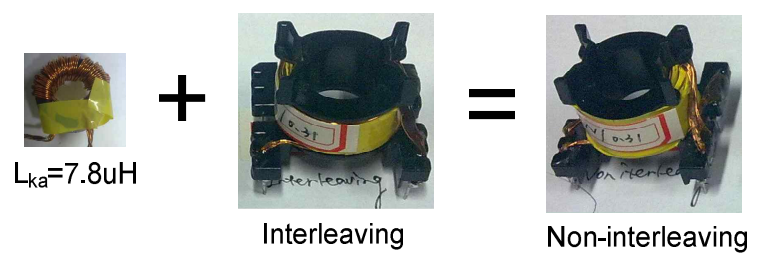

Fig. 17. External inductor $\mathrm{L}_{\mathrm{ka}}$ to balance the leakage inductance.

\section{EXPERIMENTAL VERIFICATION}

To verify the analysis results with the proposed method, four transformers (shown in Fig. 16) are built with structures given in Fig. 12(a) and Fig. 12(c). The detailed parameters and current excitation are given in Table 2 . The primary side winding of the four transformers has 48 turns so that the core losses are minimized when compared to the copper losses. To simplify the difficulties of measuring the winding loss of a power transformer, an indirect loss comparison method is adopted in this paper.

All these transformers are dropped in a $16 \mathrm{~V} / 4 \mathrm{~A}$ Quasi-Resonant flyback converter. By keeping the other circuit parameters and operations almost the same, the power loss of the whole converter with a different transformer is measured (by measuring the input power and the output power). This means that the power loss of the prototype with a different transformer is mainly caused by the transformer itself. Furthermore, since the AC magnetic flux density and the switching frequency are kept the same, the core loss is almost unchanged. Therefore, the measured power loss difference is mainly caused by the transformer winding loss, which can be compared with the calculated winding loss difference to verify the effectiveness of the proposed analysis method.

Since the leakage inductance is heavily affected by the winding structure, it also affects the circuit operation and the clamp circuit power loss. To avoid the influence of the leakage inductance on the power loss, an extra $7.8 \mathrm{uH}$ inductor $\mathrm{L}_{\mathrm{ka}}$ with very low power loss is connected in series with the interleaving transformers primary side winding to make the four transformers have the same leakage inductance, as shown in Fig. 17. Thus the clamp circuit and the circuit operation conditions can almost be the same, which makes the comparison more reasonable.

The measured total power losses of the converter with four transformers at two operating frequencies $(39 \mathrm{kHz}$ and 67 $\mathrm{kHz}$ ) are shown in Fig. 18. They are compared with the 
calculated results using the proposed method. Since the total power loss of the experimental prototype is much larger than the transformer winding loss, to make the comparison more clear in the figure, a power loss offset is introduced to roughly exclude the other power loss. In Fig. 18, the offset is used to make the measured loss with transformer $\mathrm{T} 1$ the same as the calculated one. The offset is $6.6 \mathrm{~W}$ for $39 \mathrm{kHz}$ operation and $5.5 \mathrm{~W}$ for $67 \mathrm{kHz}$ operation. It is only necessary to check that the loss difference and the offset used to exclude the other power loss will not affect the power loss difference.

As shown in Fig. 18(a), with a $39 \mathrm{kHz}$ switching frequency, the calculated winding loss difference for the interleaving structure with $\mathrm{T} 1$ and $\mathrm{T} 2$ is $0.23 \mathrm{~W}$. The measured loss difference is $0.26 \mathrm{~W}$ based on the prototype with $\mathrm{T} 1$ and $\mathrm{T} 2$. The calculated and measured power loss difference for the non-interleaving structure with $\mathrm{T} 3$ and $\mathrm{T} 4$ is $0.17 \mathrm{~W}$ and $0.16 \mathrm{~W}$, respectively. The measured power loss difference with a $67 \mathrm{kHz}$ switching frequency also matches the calculated one, as shown in Fig. 18(b). Based on the experimental results shown above, in the non-interleaving structure, the winding loss with a thicker wire $(D=0.31 \mathrm{~mm})$ is higher than that with a smaller wire $(D=0.21 \mathrm{~mm})$ even though the diameters of both wires are well below the skin depth. This means that with fine winding optimization, a lower winding conduction loss can be achieved with less copper. As expected, the interleaving structure can reduce the winding conduction loss in a flyback type transformer without considering the benefit from the reduced leakage inductance.

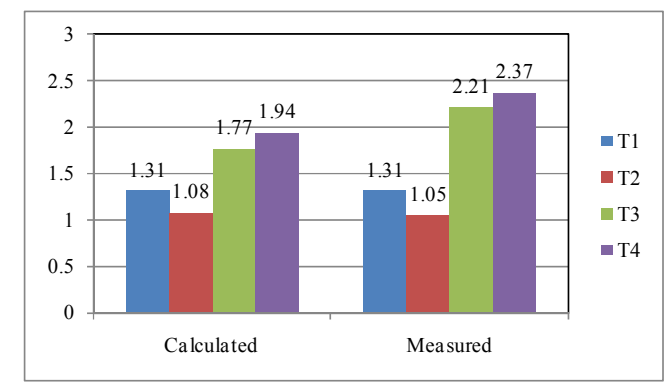

(a) $39 \mathrm{kHz}$ switching frequency.

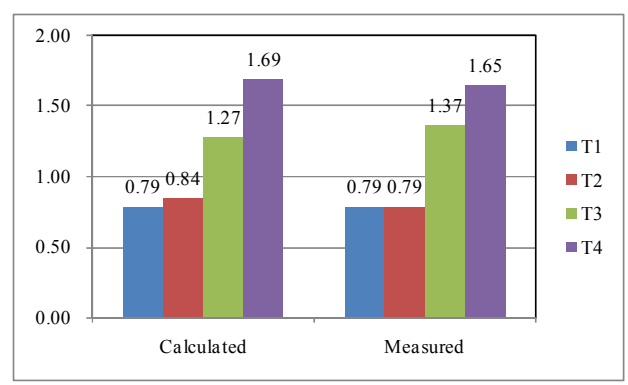

(b) $67 \mathrm{kHz}$ switching frequency.

Fig. 18. Power loss difference with different transformers.
TABLE II

Measured Key Parameters of the Four Transformers

\begin{tabular}{|c|c|c|c|c|}
\hline & $\mathrm{T} 1$ & $\mathrm{~T} 2$ & $\mathrm{~T} 3$ & $\mathrm{~T} 4$ \\
\hline $\begin{array}{c}\text { Wire } \\
\text { Diameter(mm) }\end{array}$ & 0.21 & 0.31 & 0.21 & 0.31 \\
\hline $\mathrm{Lm}(\mathrm{uH})$ & 412.5 & 412.7 & 412.5 & 412.8 \\
\hline $\mathrm{Lk}(\mathrm{uH}) @ 39 \mathrm{kHz}$ & 2.0 & 1.97 & 10.67 & 10.68 \\
\hline Lk(uH)@67kHz & 1.8 & 1.81 & 9.97 & 9.95 \\
\hline Winding Structure & pspspsps & pspspsps & ppppssss & ppppssss \\
\hline $\begin{array}{c}\text { Current Excitation } \\
(39 \mathrm{kHz})\end{array}$ & $\begin{array}{c}\mathrm{D}_{\mathrm{l}}=0.488, \mathrm{D}_{2}=0.468, \mathrm{I}_{\mathrm{p}}=3.03 \mathrm{~A}, \mathrm{I}_{\mathrm{s}}=18.18 \mathrm{~A} \\
\mathrm{D}_{\mathrm{r}}=0.005(\mathrm{pspspsps}), \mathrm{D}_{\mathrm{r}}=0.01(\mathrm{ppppssss})\end{array}$ \\
\hline $\begin{array}{c}\text { Current Excitation } \\
(67 \mathrm{kHz})\end{array}$ & $\begin{array}{c}\mathrm{D}_{1}=0.302, \mathrm{D}_{2}=0.625, \mathrm{I}_{\mathrm{p}}=2.23 \mathrm{~A}, \mathrm{I}_{\mathrm{s}}=13.38 \mathrm{~A} \\
\mathrm{D}_{\mathrm{r}}=0.012(\mathrm{pspspsps}), \mathrm{D}_{\mathrm{r}}=0.02(\mathrm{ppppssss})\end{array}$ \\
\hline
\end{tabular}

\section{CONCLUSION}

The winding loss analysis of a flyback transformer under DCM operation is difficult and ambiguous because the primary side current and the secondary side current differ both in shape and phase. In frequency domain analysis, the phase shift between the winding currents results in a 2-D field outside the air gaps, which makes the traditional 1-D model not directly applicable. First, this paper analyzed the phase shift of the winding currents in a flyback transformer and its relationship to the gap fringing field effect. Based on this analysis, the phase shift of high order harmonics ( $i \geq 2)$ is very close to $180^{\circ}$, and the fringing field is negligible. Therefore, the 1-D model can still be effective with proper consideration of the first order harmonic. Then a simplified 2-D analytical model for winding loss analysis of the flyback transformer is proposed in this paper. By simplifying the fringing field with proper assumptions, the model has an acceptable accuracy and simple form that is similar to the conventional 1-D model. The simplified analytical model is applied to analyze the winding loss of a flyback transformer with different structures. This is then verified by FEA simulation and experimental results.

\section{APPENDIX A \\ FOURIER DECOMPOSITION}

With the current excitation shown in Fig.1(b), the amplitude and phase shift of each winding current at the $i$-th harmonic are derived in (A1)-(A9).

Using the Fourier decomposition method, the amplitude and phase angle of the primary side $i$-th harmonic current can be expressed as:

$$
\begin{gathered}
I_{p}(i)=\sqrt{a_{p}(i)^{2}+b_{p}(i)^{2}} \\
\varphi_{p}(i)=\tan ^{-1}\left[\frac{b_{p}(i)}{a_{p}(i)}\right]+u_{p} \pi
\end{gathered}
$$

where $a_{p}(i)$ and $b_{p}(i)$ are the real and imaginary part of the 
primary current at the $i$-th harmonic; $u_{p}=0$ if $b_{p}(i)>0$ and $u_{p}=1$ if $b_{p}(i)<0$.

The amplitude and phase angle of the secondary side $i$-th harmonic current can also be expressed as:

$$
\begin{gathered}
I_{s}(i)=\sqrt{a_{s}(i)^{2}+b_{s}(i)^{2}} \\
\varphi_{s}(i)=\tan ^{-1}\left[\frac{b_{s}(i)}{a_{s}(i)}\right]+u_{s} \pi
\end{gathered}
$$

where $a_{s}(i)$ and $b_{s}(i)$ are the real and imaginary part of the secondary current at the i-th harmonic; $u_{s}=0$ if $b_{s}(i)>0$ and $u_{s}=1$ if $b_{s}(i)<0$.

The coefficients of the Fourier transform $a_{p}(i), b_{p}(i), a_{s}(i)$, $b_{s}(i)$ are given as:

$$
\begin{aligned}
& a_{p}(i)=\frac{2 I_{p}}{2 \pi i\left[2 \pi i \frac{D_{r}}{2}\right]}\left[\sin \left(2 \pi i\left(D_{1}+\frac{D_{r}}{2}\right)\right)\right] \sin \left(2 \pi i \frac{D_{r}}{2}\right) \\
& +\frac{2 I_{p}}{(2 \pi i)^{2} D_{1}}\left[\cos \left(2 \pi i D_{1}\right)-1\right] \\
& b_{p}(i)=\frac{-2 I_{p}}{2 \pi i\left[2 \pi i \frac{D_{r}}{2}\right]}\left[\cos \left(2 \pi i\left(D_{1}+\frac{D_{r}}{2}\right)\right)\right] \sin \left(2 \pi i \frac{D_{r}}{2}\right) \\
& +\frac{2 I_{p}}{(2 \pi i)^{2} D_{1}}\left[\sin \left(2 \pi i D_{1}\right)-1\right] \\
& a_{s}(i)=\frac{-2 I_{s}}{2 \pi i\left[2 \pi i \frac{D_{r}}{2}\right]}\left[\sin \left(2 \pi i\left(D_{1}+\frac{D_{r}}{2}\right)\right)\right] \sin \left(2 \pi i \frac{D_{r}}{2}\right) \\
& +\frac{-2 I_{s}}{(2 \pi i)^{2} D_{1}}\left[\cos \left(2 \pi i\left(D_{1}+D_{r}+D_{2}\right)\right)-\cos \left(2 \pi i\left(D_{1}+D_{r}\right)\right)\right] \\
& b_{s}(i)=\frac{2 I_{s}}{2 \pi i\left[2 \pi i \frac{D_{r}}{2}\right]}\left[\cos \left(2 \pi i\left(D_{1}+\frac{D_{r}}{2}\right)\right)\right] \sin \left(2 \pi i \frac{D_{r}}{2}\right) \\
& +\frac{-2 I_{s}}{(2 \pi i)^{2} D_{1}}\left[\sin \left(2 \pi i\left(D_{1}+D_{r}+D_{2}\right)\right)-\sin \left(2 \pi i\left(D_{1}+D_{r}\right)\right)\right]
\end{aligned}
$$

Then the phase shift between the two winding currents at the $i$-th harmonic can be derived based on (A2) and (A4), which is given in (A9).

$$
\begin{aligned}
& \varphi_{s}(i)-\varphi_{p}(i) \\
& \left.=\tan ^{-1}\left[\frac{\frac{\sin \left(2 \pi i \frac{D_{r}}{2}\right)}{\left[2 \pi i \frac{D_{r}}{2}\right]}\left[\cos \left(2 \pi i\left(D_{1}+\frac{D_{r}}{2}\right)\right)\right]+\frac{-1}{2 \pi i D_{1}}\left[\sin \left(2 \pi i\left(D_{1}+D_{r}+D_{2}\right)\right)-\sin \left(2 \pi i\left(D_{1}+D_{r}\right)\right)\right]}{\frac{-\sin \left(2 \pi i \frac{D_{r}}{2}\right)}{\left[2 \pi i \frac{D_{r}}{2}\right]}\left[\sin \left(2 \pi i\left(D_{1}+\frac{D_{r}}{2}\right)\right)\right]+\frac{-1}{2 \pi i D_{1}}\left[\cos \left(2 \pi i\left(D_{1}+D_{r}+D_{2}\right)\right)-\cos \left(2 \pi i\left(D_{1}+D_{r}\right)\right)\right]}\right]\right] \\
& +u_{s} \pi-\tan ^{-1}\left[\frac{\frac{-\sin \left(2 \pi i \frac{D_{r}}{2}\right)}{\left[2 \pi i \frac{D_{r}}{2}\right]}\left[\cos \left(2 \pi i\left(D_{1}+\frac{D_{r}}{2}\right)\right)\right]+\frac{1}{2 \pi i D !}\left[\sin \left(2 \pi i D_{1}\right)-1\right]}{\frac{\sin \left(2 \pi i \frac{D_{r}}{2}\right)}{\left[2 \pi i \frac{D_{r}}{2}\right]}\left[\sin \left(2 \pi i\left(D_{1}+\frac{D_{r}}{2}\right)\right)\right]+\frac{1}{2 \pi i D 1}\left[\cos \left(2 \pi i D_{1}\right)-1\right]}\right]-u_{p} \pi
\end{aligned}
$$

From (A9), the $i$-th harmonic phase shift of the two winding currents varies with the duty cycle. It is neither fixed at $0^{\circ}$ as an inductor, nor fixed at $180^{\circ}$ as a traditional transformer.

In order to get a much clearer insight into the current phase shift given in (A9), the expression can be further simplified based on the couple reasonable approximation.

From (A5) to (A8), the second item of $a_{p}(i), b_{p}(i)$ and $a_{s}(i)$, $b_{s}(i)$ can be neglected for high order harmonics $(i \geq 2)$ since its value is the reverse proportional of $i^{2}$. In addition, $D_{r}$ is very small when compared with the switching period, and the following is obtained:

$$
\lim _{D r \rightarrow 0} \frac{\sin \left(2 \pi i \frac{D_{r}}{2}\right)}{2 \pi i \frac{D_{r}}{2}}=1
$$

Based on the approximation given above, (A.9) can be further simplified as:

$$
\begin{aligned}
& \varphi_{s}(i)-\varphi_{p}(i) \approx \tan ^{-1}\left[\left[\frac{\left[\cos \left(2 \pi i\left(D_{1}+\frac{D_{r}}{2}\right)\right)\right]}{-\left[\sin \left(2 \pi i\left(D_{1}+\frac{D_{r}}{2}\right)\right)\right]}\right]\right] \\
& +u_{s} \pi-\tan ^{-1}\left[\frac{-\left[\cos \left(2 \pi i\left(D_{1}+\frac{D_{r}}{2}\right)\right)\right]}{\left[\sin \left(2 \pi i\left(D_{1}+\frac{D_{r}}{2}\right)\right)\right]}\right]-u_{p} \pi \mid i \geq 2
\end{aligned}
$$

With $b_{p}(i) \cdot b_{s}(i)<0$ the following is obtained: $u_{s}=0$ and $u_{p}=1$, or $u_{s}=1$ and $u_{p}=0$. Then the phase shift at higher harmonic $(i \geq 2)$ is given in (A.12).

$$
\varphi_{s}(i)-\varphi_{p}(i) \approx \pi \mid i \geq 2
$$

From (A.12), it is clear that the phase shift of high order harmonics is theoretically close to $180^{\circ}$.

\section{APPENDIX B \\ EXAMPLE}

This part provides a detailed step by step example of how to apply the proposed method to calculate the winding loss in a flyback transformer. Taking the interleaving structure as an example, the detailed parameters of the core and winding structure are shown in Fig. 12 (a) and Table 1. It uses AWG32 $(0.21 \mathrm{~mm})$ wire, the switching frequency is $100 \mathrm{kHz}$, and the skin depth for the copper at this frequency is $0.209 \mathrm{~mm}$. Hence the diameter of the wire is close to the skin depth at this frequency.

Step1: Calculate the amplitude and phase shift of each current harmonic in each layer using the Fourier decomposition method given in Appendix A. Table 3 
summaries the current harmonic amplitude and phase shift from the dc component to the $10^{\text {th }}$ harmonic in each layer.

Step2: Calculate the averaged $y$ component of the gap fringing field at the $1^{\text {st }}$ harmonic at the layer next to the gap.

The distance from gap to the adjacent layer is important to reduce the gap fringing field with a given phase shift. In this example, $x_{c}(1,0)_{k}$ is $(1.15+0.21 / 2) \mathrm{mm}$ considering the bobbin thickness and the wire diameter. The total gap length is $2 \times 0.4 \mathrm{~mm}$, and then the averaged $y$ component of the gap fringing field can be calculated by (5) in layer $(1,0)$, $\left|\overline{H_{g y}(1,0)_{1}}\right|=1.7434 \times 10^{3} \mathrm{~A} / \mathrm{m}$.

Step3: Get a complex type magnetic $\mathrm{H}$ field at each harmonic at the left/right side of each layer according to (8). The total primary layers and the secondary layers are 4 , i.e. $\mathrm{m}_{\mathrm{p}}=4$ and $\mathrm{m}_{\mathrm{s}}=4$. For example, the $1^{\mathrm{st}}$ harmonic magnetic field at the left side of layer $(1,0)$ is $\mathrm{H}_{\mathrm{y}}(0,0)_{1}=$ $\left(3.9118 \times 10^{3}-5.5978 \mathrm{j} \times 10^{3}\right) \mathrm{A} / \mathrm{m}$. In addition, the magnetic field at the right side of layer $(1,0)$ is $\mathrm{H}_{\mathrm{y}}(1,0)_{1}=\left(2.3831 \times 10^{3}\right.$ $\left.5.5978 \mathrm{j} \times 10^{3}\right) \mathrm{A} / \mathrm{m}$, and $\left|\mathrm{H}_{\mathrm{y}}(1,0)_{1}\right|=6.08 \times 10^{3} \mathrm{~A} / \mathrm{m}$. By comparing the averaged $y$ component of the fringing field $\overline{H_{g y}(1,0)_{1}}$ with the 1-D magnetic field at the right side of layer $(1,0)\left|\mathrm{H}_{\mathrm{y}}(1,0)_{1}\right|$, the $y$ component of the fringing field can be neglected and only its $x$ component, which is orthogonal to the 1-D magnetic field, needs to be considered.

Step4: Calculate the power loss caused by the proximity effect in each layer.

After the magnetic field around each layer is obtained, the loss at each layer can be derived by using (11)-(14). The proximity effect loss in layer $(1,0)$ of the $1^{\text {st }}$ harmonic can be obtained as:

$$
P_{y}(1,0)_{1}=b_{w} \cdot l(1,0) \cdot Q_{y}(1,0)_{1}=0.1271 \mathrm{~W}
$$

The proximity effect loss of the other layers as well as the other harmonics could also be calculated in the same way.

Step5: Calculate the power loss caused by the $x$ component of the fringing field.

The fringing field loss can then be calculated from (6) and (7). As mentioned before, it is reasonable to consider only the layers next to the gap since the fringing filed decays quickly with distance. The fringing loss of the two layers next to the center gap of the $1^{\text {st }}$ harmonic is given as:

$$
\begin{aligned}
& P_{g}(1,0)_{1}=b_{w} \cdot l(1,0) \cdot Q_{g c}(1,0)_{1}=0.0201 \mathrm{~W} \\
& P_{g}(1,1)_{1}=b_{w} \cdot l(1,1) \cdot Q_{g c}(1,1)_{1}=0.0133 \mathrm{~W}
\end{aligned}
$$

And the loss of the layers next to the side gap can also be calculated using the same method, which is given as:

$$
\begin{aligned}
& P_{g}(4,3)_{1}=b_{w} \cdot l(4,3) \cdot Q_{g s}(4,3)_{1}=0.0022 \mathrm{~W} \\
& P_{g}(4,4)_{1}=b_{w} \cdot l(4,4) \cdot Q_{g s}(4,4)_{1}=0.0026 \mathrm{~W}
\end{aligned}
$$

Step6: Calculate the total loss by adding the proximity and the fringing field losses at each harmonic. Table 4 lists the calculated losses at each harmonic using the proposed method.
TABLE III

Current Harmonic Amplitude and Phase Shift

\begin{tabular}{|c|c|c|c|}
\hline harmonics & $I_{p}(i)$ & $I_{s}(i)$ & Phase shift \\
\hline $\mathrm{i}=0$ & 18.1072 & 17.3802 & 0 \\
\hline $\mathrm{i}=1$ & 13.7738 & 13.5179 & 248.5189 \\
\hline $\mathrm{i}=2$ & 5.925 & 6.1726 & -179.48 \\
\hline $\mathrm{i}=3$ & 3.9256 & 3.7699 & 204.7751 \\
\hline $\mathrm{i}=4$ & 2.957 & 3.0715 & -178.985 \\
\hline $\mathrm{i}=5$ & 2.3148 & 2.2269 & 194.0526 \\
\hline $\mathrm{i}=6$ & 1.965 & 2.0317 & -178.5388 \\
\hline $\mathrm{i}=7$ & 1.6395 & 1.5868 & 189.1155 \\
\hline $\mathrm{i}=8$ & 1.4657 & 1.5076 & -178.1627 \\
\hline $\mathrm{i}=9$ & 1.2669 & 1.2349 & 186.236 \\
\hline $\mathrm{i}=10$ & 1.1661 & 1.1904 & -177.8747 \\
\hline
\end{tabular}

\section{TABLE IV}

CAlculated Power Loss at EACH HaRmonic

\begin{tabular}{|c|c|}
\hline harmonics & Loss(W) \\
\hline $\mathrm{i}=0$ & 0.4839 \\
\hline $\mathrm{i}=1$ & 0.7982 \\
\hline $\mathrm{i}=2$ & 0.1156 \\
\hline $\mathrm{i}=3$ & 0.0723 \\
\hline $\mathrm{i}=4$ & 0.0348 \\
\hline $\mathrm{i}=5$ & 0.0262 \\
\hline $\mathrm{i}=6$ & 0.0188 \\
\hline $\mathrm{i}=7$ & 0.01757 \\
\hline $\mathrm{i}=8$ & 0.00873 \\
\hline $\mathrm{i}=9$ & 0.009 \\
\hline $\mathrm{i}=10$ & 0.0088 \\
\hline
\end{tabular}

To achieve good accuracy and reasonable calculation efforts, the harmonics included in the loss calculation can range from 10 to 15 . In this example, the harmonics up to 10 $(\mathrm{i}=10)$ are summed up. The total loss is $1.5939 \mathrm{~W}$. When compared to the simulated result of $1.573 \mathrm{~W}$, the error is around $1.32 \%$.

\section{REFERENCES}

[1] P.L. Dowell, "Effects of eddy currents in transformer windings", Proc. of the IEEE, Vol. 113, pp. 1387-1394, Aug. 1966.

[2] M. P. Perry, "Multiple layer series connected winding design for minimum losses," IEEE Trans. Power Electron., Vol. PAS-98, No.1, pp. 116-123, Jan. 1979.

[3] A.M. Urling, V.A. Niemela, G.R. Skutt, T.G. Wilson, "Characterizing high-frequency effects in transformer windings-a guide to several significant articles," in Proc. IEEE. APEC, pp. 373-385, 1989.

[4] J. A. Ferreira, "Improved analytical modeling of conductive losses in magnetic components," IEEE Trans. Power Electron., Vol. 9, No. 1, pp. 127-131, Jan. 1994.

[5] X. Nan and C. R. Sullivan," An Improved Calculation of Proximity-Effect Loss in High-Frequency Windings of Round Conductors," in Proc. IEEE PESC, pp. 853-860, 2003. 
[6] P. S. Venkatraman, "Winding eddy current losses in switch mode power transformers due to rectangular wave currents," in Proc. of Powercon 11, Sec. A, pp. 1-11, 1984.

[7] B. Carsten, "High frequency conductor losses in switch mode magnetics," in Proc. High-frequency Power Converter Conf. Record, pp. 155-176, May 1986.

[8] W. G. Hurley, E. Gath, and J. G. Breslin, "Optimizing the AC resistance of multilayer transformer windings with arbitrary current waveforms," IEEE Trans. Power Electron., Vol. 15, No. 2, pp. 369-376, Mar. 2000.

[9] R. Prieto, J.A. Cobos, O.Garcia, P. Alou and J. Uceda, "High frequency resistance in flyback type transformers," in Proc. IEEE APEC, pp. 714-719, 2000.

[10] D. Lavers, E. Lavers," Waveform Dependent Switching Losses in Flyback Transformer Foil Windings," in Proc. IEEE APEC, pp. 116-112, 2002.

[11] J. M. Lopera, M. J. Prieto, F. Nuno, A. M. Pernia, and J. Sebastian, "A quick way to determine the optimum layer size and their disposition in magnetic structures," in Proc. IEEE PESC, pp. 1150-1156, 1997.

[12] C. R. Sullivan, "Computationally efficient winding loss calculation with multiple windings, arbitrary waveforms, and two- or three dimensional field geometry," IEEE Trans. Power Electron., Vol. 16, No. 1, pp.142-150, Jan. 2001.

[13] C. R. Sullivan, T. Abdallah, "Optimization of a flyback transformer winding considering two-dimensional field effects, cost and loss," in Proc. IEEE APEC, pp. 1-7, 2001.

[14] J. H. Spreen, "Electrical terminal representation of conductor loss in transformers", IEEE Trans. Power Electron., Vol. 5, No. 4, pp. 424-429, Oct. 1990.

[15] J. Vandelac and P. D. Ziogas, "A novel approach for minimizing high frequency transformer copper losses," IEEE Trans. Power Electron., Vol.3, No. 3, pp. 166-176, Jul. 1988.

[16] Z. Y. Lu and W. Chen "Novel Winding Loss Analytical Model of Flyback Transformer," in Proc. IEEE PESC, pp.1213-1218, 2006.

[17] Rudy Severns, "Additional losses in high frequency magnetics due to non ideal field distributions", in Proc. IEEE APEC., pp. 333-338, 1992

[18] P. Wallmeier, N. Frohleke, and H. Grotstollen, "Improved analytical modeling of conductive losses in gapped high-frequency inductors", IEEE Trans. Ind. Appl., Vol. 37, No. 4, pp. 558-567, Jul. 2001.

[19] Jiankun $\mathrm{Hu}$ and C. R. Sullivan, "AC resistance of planar power inductors and the quasi-distributed gap technique", IEEE Trans. Power Electron., Vol. 16, No. 4, pp. 558-567, Jul. 2001

[20] W. Chen, J. He, H. L. Luo,H. Hu, and C. Wen, "Winding loss analysis and new air-gap arrangement for high-frequency inductor," in Proc. IEEE PESC, pp. 2084-2089, 2001.

[21] X. K. Mao, W. Chen, and Y. X. Li, "Winding Loss Mechanism Analysis and Design for New Structure High-Frequency Gapped Inductor," IEEE Trans. Magnetics, Vol.41, No. 10, pp. 4036-4038, Oct 2005.

[22] F. Robert, "A Theoretical Discussion About the Layer Copper Factor Used in Winding Losses Calculation," IEEE Trans. Magnetics, Vol. 38, No. 5, pp. 3177-3179, Sep. 2002.

[23] W. A. Roshen, "Fringing field formulas and winding loss due to an air gap," IEEE Trans. Magnetics, Vol. 43, No. 8, pp. 3387-3394, Aug. 2007.

[24] T. Komma and H. Gueldner, "The effect of different air-gap positions on the winding losses of modern planar ferrite cores in switch mode power supplies," in Proc. SPEEDAM, pp.632-637, 2008.

[25] P. Wallmeier, N. Frohleke, and H. Grotstollen, "Automated optimization of high frequency inductors," in Proc. IEEE IECON, pp. 342-347, 1998.

[26] P. Wallmeier and H. Grotstollen, "Magnetic shielding applied to high frequency inductors," in Proc. IEEE IAS, pp.1131-1138, 1997

[27] E. C. Snelling, Soft Ferrites: Properties and Applications, 2nd ed. London, U.K.: Butterworth, 1988.

[28] D. Murthy-Bellur and M. K. Kazimierczuk, "Winding losses caused by harmonics in high frequency transformers for pulse-width modulated DC-DC converters in discontinuous conduction mode," IET Power Electronics, Vol. 3, No.5, pp. 804-817, Sep. 2010.

[29] D. Murthy-Bellur, N. Kondrath, and M. K. Kazimierczuk, "Transformer winding loss caused by skin and proximity effects including harmonics in PWM DC-DC flyback converter for continuous conduction mode," IET Power Electronics, Vol. 4, No. 4, pp. 363-373, Apr. 2011.

[30] D. A. Nagarajan, D. Murthy-Bellur, and M. K. Kazimierczuk, "Harmonic winding losses in the transformer of a forward pulse width modulated dc-dc converter for continuous conduction mode," IET Power Electronics, Vol. 5, No. 2, pp. 221-236, Feb. 2012.

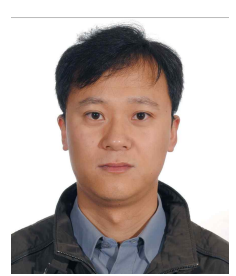

Junming Zhang received his B.S., M.S. and $\mathrm{Ph} . \mathrm{D}$. in Electrical Engineering from Zhejiang University, Hangzhou, China, in 1996, 2000 and 2004, respectively. He is currently an Associate Professor in the College of Electrical Engineering, Zhejiang University. His current research interests include power electronics system integrations, power management, high performance DC/DC converters and inverters.

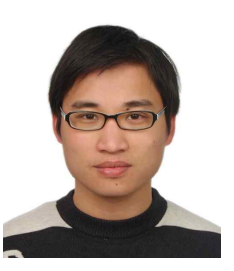

Wei Yuan received his B.S. and M.S. in Electrical Engineering from Zhejiang University, Hangzhou, China, in 2007 and 2010 , respectively. $\mathrm{He}$ is currently with Monolithic Power Systems (MPS), Hangzhou, China, as a System Engineer. His current research interests include low voltage high current conversion techniques, modeling and control of converters, and high frequency magnetic systems.

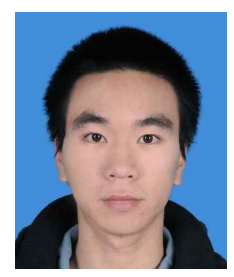

Hulong Zeng was born in Guangdong, China, in 1988. He received his B.S. in Electrical Engineering from Zhejiang University, Hangzhou, China, in 2010, where he is currently pursuing his M.S. in Electrical Engineering. His current research interests include power management and $\mathrm{AC} / \mathrm{DC}$ power converters. 


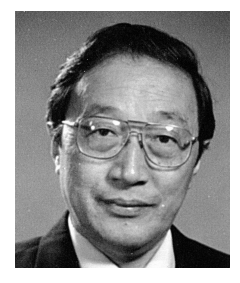

Zhaoming Qian received his B.S. in Radio Engineering from Electrical Engineering Department of Zhejiang University, Hangzhou, China in 1961. He received his Ph.D. in Applied Science from the Catholic University of Leuven and the Interuniversity Microelectronics Center (IMEC), Leuven, Belgium, in 1989. Since 1961, he has been teaching and doing research on electronics and power electronics at Zhejiang University. He was promoted to a Professor of the Electrical Engineering Department of Zhejiang University, in 1992. His current research interests include power electronics and its industrial applications, power electronic system integrations, EMC in power electronic systems, etc. He has published one book on EMC design and more than 200 papers. 